\title{
Role of conservative management in traumatic aortic injury: Comparison of long-term results of conservative, surgical, and endovascular treatment
}

\author{
Victor X. Mosquera, MD, ${ }^{a}$ Milagros Marini, MD, PhD, ${ }^{\mathrm{b}}$ José M. Lopez-Perez, MD, PhD, ${ }^{\mathrm{c}}$ \\ Javier Muñiz-Garcia, MD, PhD, ${ }^{\mathrm{d}}$ José M. Herrera, MD, PhD, ${ }^{\mathrm{a}}$ Ignacio Cao, MD, ${ }^{\mathrm{b}}$ and José J. Cuenca, MD ${ }^{\mathrm{a}}$
}

Objective: The purpose of this study is to compare early and long-term results in terms of survival and cardiovascular complications of patients with acute traumatic aortic injury who were conservatively managed with patients who underwent surgical or endovascular repair.

\begin{abstract}
Methods: From January 1980 to December 2009, 66 patients with acute traumatic aortic injury were divided into 3 groups according to treatment intention at admission: 37 patients in a conservative group, 22 patients in a surgical group, and 7 patients in an endovascular group. Groups were similar with regard to gender, age, Injury Severity Score, Revised Trauma Score, and Trauma Injury Severity Score.
\end{abstract}

\begin{abstract}
Results: In-hospital mortality was $21.6 \%$ in the conservative group, $22.7 \%$ in the surgical group, and $14.3 \%$ in the endovascular group $(P=.57)$. In-hospital aortic-related complications occurred only in the conservative group. Median follow-up time was 75 months (range, 5-327 months). Conservative group survival was $75.6 \%$ at 1 year, $72.3 \%$ at 5 years, and $66.7 \%$ at 10 years. Surgical group survival remained at $77.2 \%$ at 1 , 5 , and 10 years, whereas survival in the endovascular group was $85.7 \%$ at 1 and 5 years $(P=.18)$. No patient in the surgical or endovascular group required reintervention because of aortic-related complications, whereas $37.9 \%$ of the conservative group had an aortic-related complication that required surgery or caused the patient's death during the follow-up period. Cumulative survival free from aortic-related complications in the conservative group was $93 \%$ at 1 year, $88.5 \%$ at 5 years, and $51.2 \%$ at 10 years. Cox regression confirmed the initial type of aortic lesion (hazard ratio, 2.94; $P=.002$ ) and a Trauma Score-Injury Severity Score greater than $50 \%$ on admission (hazard ratio, $1.49 ; P=.042$ ) as risk factors for the appearance of aortic-related complications. Two peaks in the complication rate of the conservative group were detected in the first week and between the first and third months after blunt thoracic trauma.
\end{abstract}

Conclusions: The advent of thoracic aortic endografting has enabled a revolution in the management of acute traumatic aortic injury in patients with multisystem trauma with a low in-hospital morbimortality. Nonoperative management may be only a therapeutic option with acceptable survival in carefully selected patients. The natural history of these patients has revealed a marked trend of late aortic-related complications developing, which may justify an endovascular repair even in some low-risk patients. (J Thorac Cardiovasc Surg 2011;142:614-21)

Blunt rupture of the thoracic aorta usually occurs in patients with multisystem trauma and has devastating consequences. ${ }^{1}$ In order of descending frequency, rupture appears most often at the level of the aortic isthmus and then at the ascending aorta, aortic arch, distal descending aorta, and abdominal aorta. ${ }^{2}$ Several mechanisms of acute traumatic aortic injury (ATAI) have been proposed: (1) shearing stress to the aorta

\footnotetext{
From the Departments of Cardiac Surgery ${ }^{\mathrm{a}}$ and Interventional Radiology, ${ }^{\mathrm{b}}$ and Intensive Care Unit, ${ }^{\mathrm{c}}$ Complejo Hospitalario Universitario de A Coruña, Spain; and Instituto Universitario de Ciencias de la Salud, ${ }^{\mathrm{d}}$ Universidad de A Coruña, Spain. Disclosures: Authors have nothing to disclose with regard to commercial support. Received for publication Aug 8,2010; revisions received Sept 29, 2010; accepted for publication Oct 24, 2010; available ahead of print Jan 27, 2011.

Address for reprints: Victor X. Mosquera, MD, Cardiac Surgery Department, Complejo Hospitalario Universitario de A Coruña, As Xubias n84, CP 15006, A Coruña, Spain (E-mail: Victor.X.Mosquera.Rodriguez@sergas.es). 0022-5223/\$36.00

Copyright $($ c 2011 by The American Association for Thoracic Surgery doi: $10.1016 /$ j.jtcvs.2010.10.044
}

during rapid deceleration; (2) compression of the aorta between sternum and thoracic spine (osseous pinch); and (3) direct load causing aortic wall strain and medial tears.,

Traditional treatment of blunt traumatic aortic rupture was early open surgical repair with graft interposition. ${ }^{5}$ However, subsequent studies suggested that some selected patients with major associated injuries could be managed safely with delayed repair, provided blood pressure and contractility were adequately controlled. ${ }^{6,7}$ Effective control of blood pressure with beta-blockers or other antihypertensive medications remains the cornerstone of safe delayed operations. Nevertheless, in some patients a definitive conservative approach and medical management are chosen. The long-term safety of conservative management and its effect on outcomes are poorly documented. Most studies focus only on in-hospital mortality and morbidity, which has led to a lack of information on the long-term outcomes of patients treated nonoperatively. 


\section{Abbreviations and Acronyms \\ ATAI = acute traumatic aortic injury \\ ICU = intensive care unit \\ ISS $=$ Injury Severity Score \\ RTS = Revised Trauma Score \\ TRISS $=$ Trauma Injury Severity Score}

This study, based on 30 years of experience, compares early and long-term results in terms of survival and cardiovascular complications of patients with ATAI who were conservatively managed and patients who underwent a surgical or an endovascular repair. To the best of our knowledge, this is the largest single-center series of definitively nonoperatively managed ATAI reported in the literature.

\section{PATIENTS AND METHODS}

From January 1980 to December 2009, 72 patients were admitted to the Complejo Hospitalario Universitario de A Coruña with ATAI. Six patients were excluded from the analysis because of deficient documentation of the time from injury to procedure or in extremis status on arrival. Data collection included age, gender, mechanism of injury, initial clinical presentation (blood pressure, Glasgow Coma Scale), Injury Severity Score (ISS), ${ }^{8} \mathrm{Ab}-$ breviated Injury Score for each body area (head, chest, abdomen, extremities), Revised Trauma Score (RTS), ${ }^{9}$ Trauma Injury Severity Score (TRISS),${ }^{10}$ method of diagnosis (computed tomography scan, angiography, transesophageal echocardiogram, magnetic resonance imaging), initial type of aortic injury (intramural hematoma without intimal tear; partial intimal tear $<10 \mathrm{~mm}$; aortic transection/circumferential tear or intimal tear $>$ $10 \mathrm{~mm}$; aneurysm/pseudoaneurysm), site of aortic injury (aortic rootascending aorta; aortic arch; aortic isthmus; mid or distal descending thoracic aorta), and type of definitive management (conservative, open repair, or endovascular repair).

An ISS of more than 50 points predicts a mortality rate of more than $50 \%$, whereas a score of more than 70 points predicts a mortality rate of approximately $100 \%{ }^{8}$ The TRISS directly predicts the expected death rate for blunt trauma. ${ }^{10}$ Patients were classified for an ISS more than 50 points and a TRISS greater than $50 \%$ mortality to determine whether these high scores, which involve a high mortality in patients with multisystem trauma, may also predict an unfavorable course in nonoperated traumatic aortic injuries.

For the purpose of statistical analysis, the initial type of aortic injury was later divided into 2 categories: minor, including intramural hematoma without intimal tear and intimal tear less than $10 \mathrm{~mm}$, or major, including transection, intimal tear more than $10 \mathrm{~mm}$, and aneurysm/pseudoaneurysm.

The patients studied were divided into 3 groups (conservative, surgical, and endovascular) according to primary intended treatment. Patients were assigned to the conservative group when the initially intended management was indefinite nonoperative therapy. The criteria for primary intended treatment had been strictly recorded and justified in every patient history chart by the multidisciplinary team who approached each patient on admission. Obviously, these criteria have not been consistent over the observation period of 30 years. The criteria of patient management were modified with the incorporation of endovascular thoracic aorta stent-grafting based on a modern risk-benefit evaluation and critical assessment of comorbidity status. Emergency $(<24 \mathrm{~h})$ endovascular aortic repair was available at our institution only since January 2003 because of the need for an in-hospital stock of thoracic aortic endografts. The flowchart in Figure 1 depicts the modification in patient management since the incorporation of emergency aortic endografting at our institution. Indeed, only 2 patients required open surgical repair since 2003, and those patients were not suitable candidates for an endovascular repair because of anatomic considerations.

The nonoperative group included patients not initially considered for surgical or endovascular intervention because of 1) patient or family decision based on religious issues (6 patients) and 2) clinical judgment (12 low-risk patients, including patients with an intramural hematoma without intimal tear or an intimal flap $<10 \mathrm{~mm}$, and 19 high-risk patients, including those with severe associated injuries, advanced age, or other severe premorbid conditions). Two patients from the conservative group required emergency surgical or endovascular treatment because of in-hospital aortic-related complications during the first 15 days of hospitalization. None of those crossover patients were assigned to the surgical or endovascular group because their initial intended treatment was conservative. The surgical group included those patients who required surgery during the 48 first hours after admission because of hemodynamic instability caused by aortic injury. The endovascular group included patients who required aortic endografting during the first 48 hours after admission because of an aortic injury in the context of blunt thoracic trauma.

The primary outcomes measured were in-hospital mortality, late mortality, and long-term survival free from aortic-related complications. Secondary outcomes measured included length of intensive care unit (ICU) and hospital stay.

\section{Conservative Treatment}

Conservative treatment consisted of strict control of both contractility and blood pressure by continuous hypotensive infusions, such as betablockers (labetalol) and arterial vasodilators (sodium nitroprusside and calcium-blocking drugs), in patients with normal or high blood pressure. Systolic blood pressure was also maintained at more than $120 \mathrm{~mm} \mathrm{Hg}$ with intensive intravenous fluid infusion. Antihypertensive therapy was changed to oral administration when the patient was hemodynamically stable. Regular radiologic follow-up was indicated after discharge with a control thoracic computed tomography scan at 1,3,6, and 12 months and then annual cardiovascular magnetic resonance scans.

\section{Open Surgical Repair}

In our series, 22 patients underwent open surgical repair of ATAI. On the basis of the time from injury to definitive aortic repair, 20 patients underwent emergency $(<24$ hours) open surgical repair and 2 patients underwent delayed ( $>24$ hours) open surgical repair. The 2 patients who underwent delayed open repair underwent operation between 48 and 72 hours after admission. In both cases the primary intended treatment was surgical repair, but it was temporarily delayed to determine the patient's neurologic prognosis.

An aortic prosthesis was used in 14 patients, and direct suturing of the injured aorta was performed in 8 patients. A left heart bypass was established in 12 patients, and cardiopulmonary bypass was selected in 2 cases. In the remaining 8 patients, surgery was performed without cardiopulmonary support by using a simple clamping technique (clamp and sew).

\section{Endovascular Stent-Graft Treatment}

Seven patients underwent emergency $(<24$ hours) endovascular repair in our series. Endovascular stent placement procedures were performed in the operating room with patients receiving general anesthesia, as we previously reported. ${ }^{11}$ Access was via the right common femoral artery in all patients. Thoracic aortic endovascular repair was performed in the first 2 patients with the Talent thoracic stent-graft and in the other 5 patients with Valiant thoracic stent-grafts (Medtronic, World Medical Manufacturing Corp, Sunrise, Fla). All patients required a single stent-graft to cover the lesion. No patient required covering of left subclavian artery. 


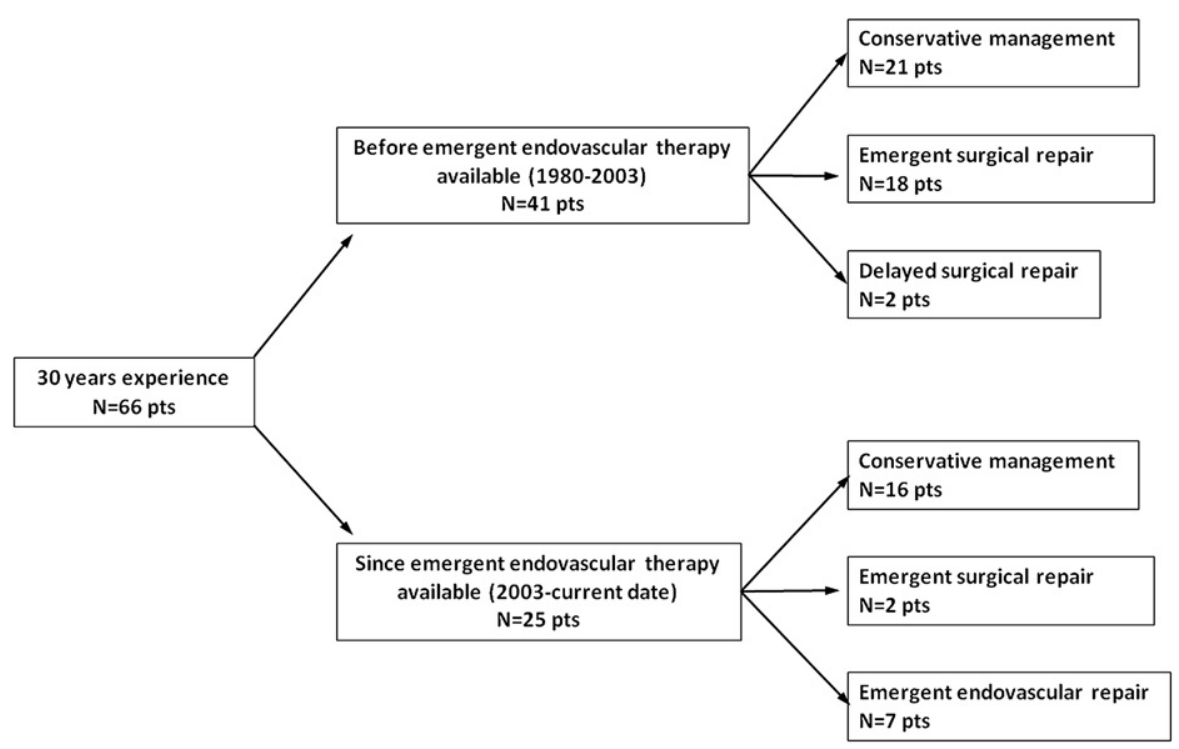

FIGURE 1. Flowchart depicts the modification in patient management since the use of emergency aortic endografting at the Complejo Hospitalario Universitario de A Coruña.

\section{Statistical Analysis}

The SPSS statistical program for Windows version 17.0 (SPSS Inc, Chicago, Ill) was used to perform data analysis. Data are expressed as mean and standard deviation or as median and range, when appropriate. Outcomes were compared among groups for the total study population, using 1-way analysis of variance (with Bonferroni adjustment) and multivariate analysis. When needed, for bivariate analysis, proportions were compared with contingency tables by means of chi-square or Fisher exact tests, and the Student $t$ test or Mann-Whitney rank-sum test was used to compare means.

Cox regression analysis was used to compare the different influence on mortality among the study groups, adjusting for sex, age, TRISS, and aortic injury type. The adjusting variables were selected according to their clinical relevance in patients with multisystem trauma and previously published literature. Adjusted hazard ratio, $95 \%$ confidence intervals, and $P$ values were derived.

Bivariate analysis was used to identify variables of potential influence in the probability of developing aortic-related complication during follow-up in the conservative group. Cox regression analysis was used to confirm or reject these variables as influencing factors in the probability of complication. Adjusted hazard ratio, $95 \%$ confidence intervals, and $P$ values were derived.
Actuarial estimates of survival were accomplished with Kaplan-Meier methods. Differences in probability of survival among the 3 groups were analyzed with the log-rank (Mantel-Cox) test.

\section{RESULTS \\ In-Hospital Results}

Most patients were male $(86.3 \%)$ with a mean age of $39.6 \pm 17.3$ years, including $21.2 \%$ of patients aged more than 55 years. Expected mortality at admission was 50\% or greater according to an ISS greater than 50 points in $25.7 \%$ of patients, whereas overall mean expected death rate calculated by TRISS was $50.9 \% \pm 24.2 \%$. A total of $31.1 \%$ of patients had at least 1 severe extrathoracic injury with AIS greater than 3. Epidemiologic and clinical characteristics of the study patients are shown in Table 1.

The study population was divided into 3 groups according to treatment intentions at admission: 37 patients in the conservative group, 22 patients in the surgical group, and 7 patients in the endovascular group. Average age was

TABLE 1. Epidemiologic and clinical characteristics in all patients and conservative and surgical groups

\begin{tabular}{|c|c|c|c|c|c|}
\hline & All patients $(n=66)$ & Conservative group $(n=37)$ & Surgical group $(n=22)$ & Endovascular group $(n=7)$ & $P$ value \\
\hline Male & $57(86.3 \%)$ & $34(91.9 \%)$ & $16(68.1 \%)$ & $7(100 \%)$ & .18 \\
\hline Age $($ mean $\pm \mathrm{SD})$ & $39.6 \pm 17.3$ & $44.2 \pm 19.3$ & $30.8 \pm 9.8$ & $44.9 \pm 13.8$ & .008 \\
\hline Age $>55$ y & $14(21.2 \%)$ & $13(35.1 \%)$ & 0 & $1(14.2 \%)$ & .004 \\
\hline ISS (mean \pm SD) & $43.4 \pm 16.4$ & $39.3 \pm 18.5$ & $40 \pm 13.1$ & $46.1 \pm 14.9$ & .62 \\
\hline $\mathrm{RTS}($ mean $\pm \mathrm{SD})$ & $6.3 \pm 1.6$ & $6.2 \pm 1.7$ & $6.3 \pm 1.5$ & $6.4 \pm 1.8$ & .92 \\
\hline TRISS (mean \pm SD) & $55.9 \pm 24.2$ & $50.1 \pm 23.5$ & $60.1 \pm 21.2$ & $52 \pm 23.1$ & .44 \\
\hline Head AIS $>3$ & $19(28.8 \%)$ & $12(32.4 \%)$ & $6(27.2 \%)$ & $1(14.2 \%)$ & .48 \\
\hline Abdomen AIS $>3$ & $16(24.2 \%)$ & $8(21.6 \%)$ & $6(27.2 \%)$ & $2(28.5 \%)$ & .46 \\
\hline Extremity AIS > 3 & $25(37.8 \%)$ & $14(37.8 \%)$ & $10(45.4 \%)$ & $1(14.2 \%)$ & .43 \\
\hline
\end{tabular}

ISS, Injury Severity Score; AIS, Abbreviated Injury Score; RTS, Revised Trauma Score; TRISS, Trauma Injury Severity Score. $P$ value of proportions analysis was obtained with the chi-square test; $P$ value mean analysis corresponds to 1-way analysis of variance with Bonferroni adjustment. 
TABLE 2. Mechanisms of acute traumatic aortic injury

\begin{tabular}{lc}
\hline \multicolumn{1}{c}{ Mechanism } & No. of patients $(\%)$ \\
\hline Motor vehicle crash (frontal impact) & $35(53)$ \\
Motor vehicle crash (lateral impact) & $5(7.6)$ \\
Motorcycle & $11(16.7)$ \\
Vehicle-pedestrian & $4(6)$ \\
Fall & $8(12.1)$ \\
Crushed under weight & $3(4.5)$ \\
\hline
\end{tabular}

significantly lower in the open surgical group, and the conservative group presented a higher proportion of patients aged more than 55 years (Table 1). The 3 groups were similar with regard to gender, presence of severe extrathoracic injuries, and expected mortality calculated by ISS, RTS, and TRISS (Table 1). The cause of aortic injury was rapid deceleration from blunt thoracic trauma resulting from different mechanisms summarized in Table 2.

Most aortic injuries occurred at the isthmus level $(65.2 \%)$ followed in frequency by the mid and distal thoracic descending aorta $(24.2 \%)$. Lesion at the aortic arch appeared in $10.6 \%$ of patients. There were statistically significant differences in the types of aortic injury (intramural hematoma without intimal tear; partial intimal tear $<10$ $\mathrm{mm}$; aortic transection/intimal tear $>10 \mathrm{~mm}$; aneurysm/ pseudoaneurysm) among the 3 study groups (Table 3 ). Patients in the conservative group had a significantly smaller proportion of high-severity aortic injuries (transection/intimal tear of $>10 \mathrm{~mm}$ ) compared with patients in the surgical and endovascular groups, whereas there were no significant differences between the surgical and endovascular groups, which both presented a high proportion of high-severity aortic injuries (24.3\% in the conservative group compared with $86.4 \%$ and $85.2 \%$ in the surgical and endovascular groups, respectively, $P<.001$ ).

Overall in-hospital mortality was $18.2 \%: 21.6 \%$ in the conservative group, $22.7 \%$ in the surgical group, and $14.3 \%$ in the endovascular group. There was a clear trend toward higher in-hospital mortality in the conservative group, but it was not statistically significant $(P=.57)$. Causes of death are summarized in Table 4 . As can be observed, most overall causes of death were directly related to ATAI complications: free aortic rupture in $58.3 \%$ of deaths and mesenteric ischemia caused by distal organ malperfusion in $8.3 \%$ of deaths. Although there were no statistically significant differences among groups in all-cause mortality, all aortic-related complications and aortic-related mortality occurred in the conservative group ( $100 \%$ conservative vs $0 \%$ in both surgical and endovascular groups, $P<.001$ ).

Although the conservative group had shorter ICU and inhospital stays than the surgical and endovascular groups, these differences were not statistically significant. The overall median ICU stay length was 16 days (range, $0-123$ days). The median ICU stay length was 12 days (range, 0-61 days) in the conservative group, 14 days (range, $0-123$ days) in the surgical group, and 20 days in the endovascular group (range, $0-45$ days) $(P=.46)$. The overall median in-hospital stay length was 30 days (range, 0-228 days). The median in-hospital stay length was 26 days (range, 0-203 days) in the conservative group, 29 days (range, $0-228$ days) in the surgical group, and 30 days (range, $0-115$ days) in the endovascular group $(P=.79)$.

No patient in the surgical group presented paraplegia after the aortic repair. However, postoperative left phrenic nerve palsy occurred in 3 patients and bilateral palsy occurred in 1 patient after open surgical repair. Ischemia from femoral arterial occlusion developed in 1 patient, and femoral arterial thrombosis at the cannulation site developed in 1 patient; both had undergone open repair with left heart bypass and required surgical repair of the femoral artery. Patients treated with endografting had no neurologic or device-related complications.

\section{Long-Term Results}

After hospital discharge, clinical and imaging follow-up was available in all patients at a median of 75 months (range, 5-327 months). Overall survival estimated by the Kaplan-Meier method, including early mortality, was $81.8 \%$ at 1 year, $75.1 \%$ at 5 years, and $72.7 \%$ at 10 years. Conservative group survival was $75.6 \%$ at 1 year, $72.3 \%$ at 5 years, and $66.7 \%$ at 10 years (Figure 2). Surgical group survival remained at $77.2 \%$ at 1,5 , and 10 years (Figure 2). The endovascular group presented a survival of $85.7 \%$ at 1 and 5 years, but none of these patients have reached a 10-year follow-up yet (Figure 2). Although there is a clear trend toward a higher long-term survival in the endovascular group, no statistically significant differences in group survival were detected (log-rank test $P=.59$ ). Cox regression revealed no differences among groups after adjusting for sex, age, initial type of aortic injury, and TRISS.

After hospital discharge, 11 of the surviving 29 patients $(37.9 \%)$ in the conservative group had an aortic-related

TABLE 3. Types of aortic injury

\begin{tabular}{|c|c|c|c|c|c|}
\hline & All patients $(n=66)$ & Conservative group $(n=37)$ & Surgical group $(n=22)$ & Endovascular group $(n=7)$ & $\chi^{2}$ \\
\hline Intramural hematoma & $15(22.7 \%)$ & $15(40.5 \%)$ & 0 & 0 & \\
\hline Partial intimal tear & $14(21.2 \%)$ & $10(27.1 \%)$ & $3(13.6 \%)$ & $1(14.2 \%)$ & \\
\hline Transection & $34(51.5 \%)$ & $9(24.3 \%)$ & $19(86.4 \%)$ & $6(85.8 \%)$ & $<0.001$ \\
\hline Aneurysm/pseudoaneurysm & $3(4.5 \%)$ & $3(8.1 \%)$ & 0 & 0 & \\
\hline
\end{tabular}


TABLE 4. Distribution of causes of in-hospital mortality

\begin{tabular}{lcccc}
\hline \multicolumn{1}{c}{ Cause of in-hospital mortality } & Overall patients $(\mathbf{n}=\mathbf{1 2})$ & Conservative group $(\mathbf{n}=\mathbf{8})$ & Surgical group $(\mathbf{n}=\mathbf{3})$ & Endovascular group $(\mathbf{n}=\mathbf{1})$ \\
\hline Aortic rupture & $7(58.3 \%)$ & $7(87.5 \%)$ & 0 & 0 \\
Multiorgan failure & $2(16.6 \%)$ & 0 & $1(33.3 \%)$ & $1(100 \%)$ \\
Acute mesenteric ischemia & $1(8.3 \%)$ & $1(12.5 \%)$ & 0 & 0 \\
Acute respiratory distress syndrome & $1(8.3 \%)$ & 0 & $1(33.3 \%)$ & 0 \\
Septic shock & $1(8.3 \%)$ & 0 & $1(33.3 \%)$ & 0 \\
\hline
\end{tabular}

complication that required surgical or endovascular repair or caused the patient's death during the follow-up period. No patient from the surgical or the endovascular group required reintervention because of an aortic-related complication during the follow-up period $37.9 \%$ in conservative group vs $0 \%$ in both surgical and endovascular groups, $P=$.004). Cumulative survival free from aortic-related complications in the surviving patients of the conservative group after hospital discharge ( $\mathrm{n}=29$ patients) was $93 \%$ at 1 year, $88.5 \%$ at 5 years, and $51.2 \%$ at 10 years (Figure 3). Late aortic-related complications were 2 free aortic ruptures, which occurred between months 1 and 3 after blunt thoracic trauma and with both patients attending ambulatory rehabilitation; 1 progression of an initially localized dissection with distal perfusion impairment; and 8 cases of formation of posttraumatic pseudoaneurysm. Both free aortic ruptures and the progression of dissection led to the patient's death. All of the posttraumatic pseudoaneurysms were eccentric saccular aneurysms greater than 2 $\mathrm{cm}$ in diameter, with a total aortic size greater than $5 \mathrm{~cm}$, and all required surgical repair.

Bivariate analysis suggested sex, age, initial type of aortic lesion, TRISS greater than $50 \%$, and ISS greater than 50 points as variables of potential influence relative to the probability of developing aortic-related complications in

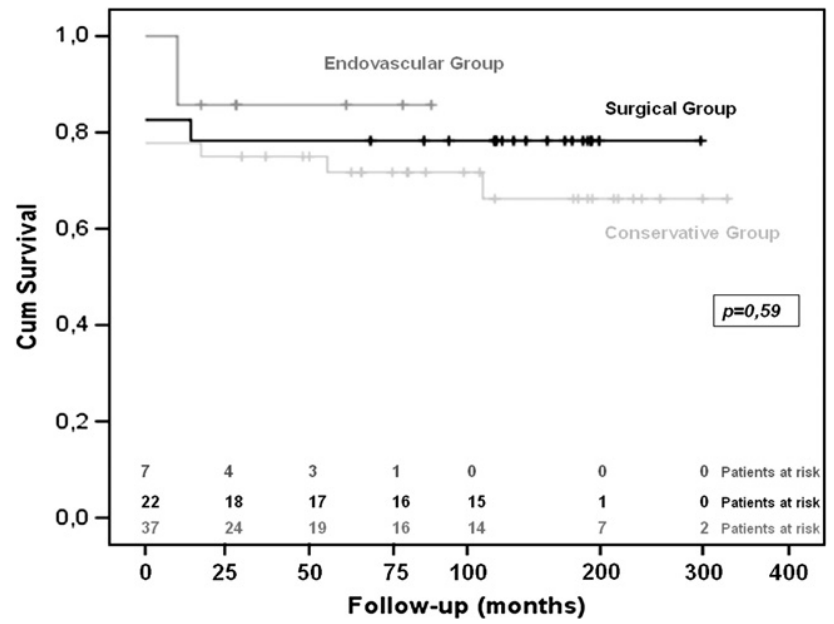

FIGURE 2. Kaplan-Meier survival curves of conservative, surgical, and endovascular groups, including in-hospital mortality. There is a clear trend toward a greater long-term survival in the endovascular group, without being statistically significant $(P=.59)$. the conservative group. Nevertheless, Cox regression only confirmed the initial type of aortic lesion (hazard ratio, $2.94 ; P=.002$ ) and TRISS greater than $50 \%$ (hazard ratio, $1.49 ; P=.042)$ on admission as risk factors for the appearance of aortic-related complications during follow-up. Indeed, all 18 patients in the uncomplicated nonoperative group presented low-risk aortic injuries (hematoma without intimal tear or intimal tear $<10 \mathrm{~mm}$ ). Moreover, ISS, RTS, and TRISS at the time of hospital admission were significantly lower in the uncomplicated nonoperative group than in those of the 11 patients in the complicated group. However, there were no significant differences in age and sex between complicated and uncomplicated nonoperative groups.

In the 18 patients of the conservative group without aortic-related complications during follow-up, 10 had complete radiographic resolution of their injuries, whereas the remaining patients had asymptomatic and radiographically stable residual injuries.

\section{DISCUSSION}

Long-term outcomes of patients with ATAI in whom a definitive nonoperative approach is selected remain obscure. With a median follow-up of 75 months, we have found

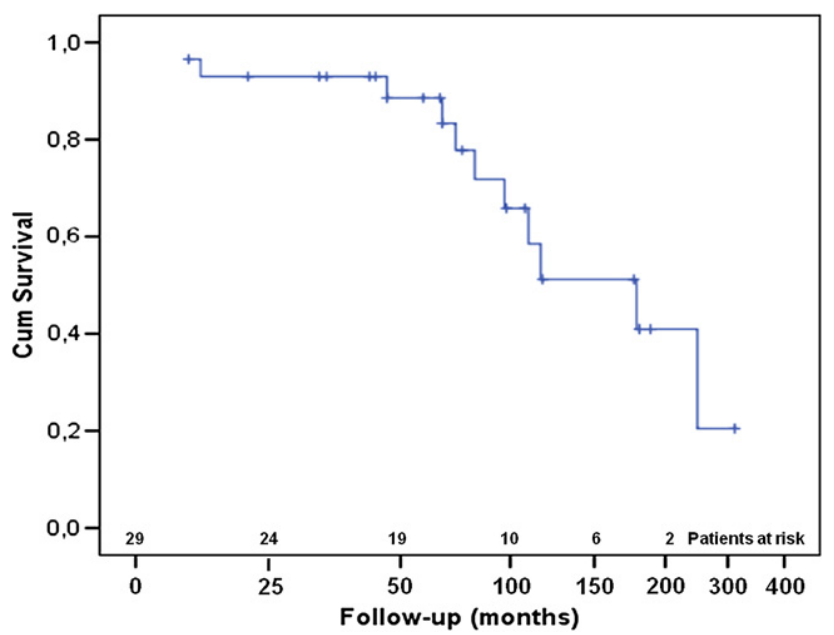

FIGURE 3. Cumulative survival free from aortic-related complications in the surviving patients of the conservative group after hospital discharge $(\mathrm{n}=29)$. 
that, although there were no significant differences in in-hospital and long-term survival among groups, 37.9\% of nonsurgically managed patients had an aortic-related complication after hospital discharge, which led to the patient's death or required surgical or endovascular repair. We also identified 2 peaks of high complication rates in the natural history of conservatively managed patients.

In the late 1950s, Parmley and colleagues ${ }^{12}$ reported a death rate at the scene of as high as $85 \%$ and a subsequent mortality rate in nonoperated survivors of $1 \%$ per hour for the first 48 hours. Those data led to the traditional emergency surgical approach to aortic injuries after blunt thoracic trauma. Moreover, surviving patients include approximately $5 \%$ who are hemodynamically unstable or deteriorate within 6 hours of admission, leading to in-hospital mortality as high as $90 \%$ or more. ${ }^{13}$

Although the traditional approach to ATAI has been emergency ( $<24$ hours) surgical repair, currently there is a trend toward delayed repair (open or endovascular). Several studies have suggested that some patients with major associated injuries, ${ }^{7}$ or even with no severe associated injuries or major comorbidities, ${ }^{14}$ can be safely managed with delayed repair, provided blood pressure and contractility are adequately controlled.

Effective control of blood pressure and contractility with beta-blockers or other antihypertensive medications remains the cornerstone for medical management of aortic injuries after blunt thoracic trauma. According to the Recommendations of the European Society of Cardiology Task Force on Aortic Dissection, ${ }^{15}$ control of blood pressure and contractility is of utmost importance in traumatic aortic dissection, regarded as a class 5 aortic dissection, to avoid progression to frank aortic rupture. Delayed repair may permit better resuscitation and performance of the procedure under more optimal conditions and perhaps reduce the risk of complications, especially paraplegia. ${ }^{16}$

Definitive nonoperative management may be chosen in patients in whom a hemodynamically stable situation is achieved with medical therapy, especially if major extrathoracic injuries are associated. In our study, in-hospital mortality was similar in the 3 groups, but with a slightly lower in-hospital death rate in the endovascular group (14.3\% in the endovascular group vs $21.6 \%$ and $22.7 \%$ in the conservative and surgical groups, respectively, $P=.57)$. In contrast, the expected mortality at hospital admission tended to be higher in the endovascular group according to the trauma scores, although this difference was not statistically significant. Indeed, all the in-hospital aortic-related complications (free aortic rupture or progression of dissection) occurred only in the conservative group and led to a switch to an endovascular or surgical management or directly to patient death.

Although it was not significant, both ICU and in-hospital stay lengths tended to be shorter in the conservative group.
We can easily explain this finding because the length of inhospital stay in patients with multisystem trauma is usually determined by the severity of the extrathoracic injuries and not by the cardiovascular injuries.

Holmes and colleagues ${ }^{17}$ reported the results of a study of 30 patients with ATAI classified in a group treated with delayed surgery or in a nonoperatively managed group with a median follow-up of 2.5 years. In that report, one third of patients from the nonoperative group died within the first 5 days, and, in contrast with our study, nonoperatively managed patients were significantly older and tended to be more severely injured according to the ISS. However, their data were clearly limited by the small numbers of patients involved. By contrast, with more than double the number of patients and a median follow-up of 6 years, our study showed that patients who underwent nonsurgical management have a greater long-term probability of aortic-related complications developing after discharge than the surgical or endovascular groups $(37.9 \%$ vs $0 \%, P=.004)$. Moreover, the long-term survival tended to be poorer in the conservative group than in the surgical and endovascular groups $(72.3 \%$ in the conservative group compared with $78.2 \%$ and $85.7 \%$ in the surgical and endovascular groups at 5 years, $P=.18$ ).

In 2002, Kepros and colleagues ${ }^{18}$ reported a mini-series of 5 patients with conservative management after ATAI in whom complete resolution of small intimal tears $(<20$ $\mathrm{mm}$ ) was documented by transesophageal echocardiography between 3 and 19 days. After a review of nonoperative series in the literature, the largest one including 19 patients, Hirose and colleagues ${ }^{19}$ concluded that nonoperative management of ATAI may be the treatment of choice in selected patients, especially those with multiple associated injuries or severe comorbidity and those with an aortic flap less than $10 \mathrm{~mm}$.

Caffarelli and colleagues ${ }^{20}$ recently reported the early outcomes of 29 patients who underwent planned nonoperative management with a survival of $97 \%$ at a median of 1.8 years (range, 0.9-7.2 years). They concluded that deliberate, nonoperative management of carefully selected patients with traumatic blunt aortic injury may be a reasonable alternative in patients with multisystem trauma. However, serial imaging and long-term outcomes (survival and aorticrelated complications) remained obscure.

Although some authors ${ }^{21}$ have reported that chronic thoracic aortic aneurysm develops in only $7 \%$ of patients with a history of untreated ATAI, we have found that the aortic injury progressed to a posttraumatic aneurysm in 8 $(27.5 \%)$ of the 29 patients in the conservative group who survived and were discharged from hospital. Furthermore, as we have previously pointed out, all of them required late surgical or endovascular repair of the aneurysm during the follow-up period.

Our experience is consistent with the literature to date in many respects, yet provides some new insights on the 
nonoperative treatment of ATAI. Long-term follow-up has been essential to determine whether nonsurgical management has had any impact on the natural history of the aortic injury. Our study detected 2 peaks in the complication rate of the nonsurgical group. The first peak occurred during the first week and mainly affected those patients with a major or borderline aortic radiologic injury. The second peak appeared between the first and third months after blunt thoracic trauma. Indeed, 2 patients from the conservative group had a critical aortic-related complication (free aortic rupture) between months 1 and 3 after thoracic trauma. Both of them presented an aortic intimal tear greater than $10 \mathrm{~mm}$.

The introduction of thoracic aorta stent-grafts has revolutionized the definitive management of ATAI. Thoracic aortic endografting for ATAI was used initially in those with high-risk multiple injuries or elderly patients, but in many centers it has now become the initial procedure of choice, even in young or low-risk patients. Advantages of endografting include avoidance of thoracotomy, single-lung ventilation, aortic crossclamping, and left heart or cardiopulmonary bypass. Endovascular aortic repair also requires considerably less time and can be done expeditiously in relatively unstable patients. A systematic review by Akowuah and colleagues ${ }^{22}$ showed that operative mortality and postoperative paraplegia rates were significantly less for thoracic endografting compared with open surgical repair (7\% vs $19 \%[P<.01]$ and $1 \%$ vs $6 \%[P<.01]$, respectively). Nevertheless, the authors stressed that long-term outcomes are poorly documented.

A multicenter, prospective study of the American Association for the Surgery of Trauma, which included 193 patients, compared outcomes of endografting in ATAI with standard operative repair. Endovascular aortic repair was associated with significantly lower mortality and fewer blood transfusions, but reported a considerable risk of serious device-related complications. ${ }^{14}$ However, a word of caution must be posed because the long-term results with endovascular repair are not known and aortic endografting may involve device-related complications such as stent collapse. ${ }^{23,24}$ In the same year, a meta-analysis of retrospective cohort studies indicated that endovascular treatment of descending thoracic aortic trauma is an alternative to open repair and associated with lower postoperative mortality and ischemic spinal cord complication rates. ${ }^{25}$ The Expert Opinion Committee of the Society of Thoracic Surgeons and the American Association of Thoracic Surgeons suggested that both acute and chronic traumatic aortic injuries be considered for treatment with endografts. ${ }^{26}$ Thoracic aortic endografting was also recommended as first-line treatment for ATAI in the ACC and AHA ACC new practice guidelines for the management and treatment of thoracic aortic disease. $^{27}$

In our series, based on a 30-year experience, severe aortic-related complications developed after hospital discharge in $37.9 \%$ of patients nonoperatively managed, especially in the period before endovascular therapy was available. The natural history of those patients would have justified an early endovascular repair in most of the conservative group. Multidetector computed tomography has a sensitivity of $96.0 \%$, specificity of $99.8 \%$, positive predictive value of $92.3 \%$, negative predictive value of $99.9 \%$, and accuracy of $99.8 \%$ in ATAI diagnosis. ${ }^{28}$ With the advent of multidetector computed tomography and in combination with the invaluable data provided by transesophageal echocardiography, especially in intubated patients, we currently perform an accurate stratification of the aortic injury according to the risk of aortic complication and perform an early endovascular repair whenever possible in all ATAIs, except in aortic intramural hematoma with no identifiable intimal tear and in small intimal tears less than 10 $\mathrm{mm}$, which are the lowest risk injuries in which indefinite delay in repair may be allowed.

\section{Limitations}

This study presents the limitations inherent to any retrospective series. The conservative group was more heterogeneous and included low-risk patients, the majority of whom could be managed safely with serial imaging (eg, intramural hematoma) and in whom one would expect a low mortality, and the most high-risk patients (eg, elderly patients, those with severe associated comorbidities), in whom we would expect a higher mortality and an increased complication rate. Nevertheless, the 3 groups were similar with regard to gender, age, presence of severe extrathoracic injuries, and expected mortality calculated by current trauma scores (ISS, RTS, and TRISS). The criterion of primary intended treatment (conservative, surgical, or endovascular management) was not consistent during the observation period of 30 years and was modified with the inclusion of technologic advances in both diagnostic and therapeutic fields, especially with the spread of thoracic aortic endografting. Although the patient population in the present study reflects the wide clinical spectrum of ATAI seen in patients with multisystem trauma, and considering that ATAI is not a common entity, the total number of patients is generally small and the present study lacks sufficient statistical power to determine with confidence some clinical relevant differences. However, the strength of this study lies in the longterm results of the largest single-center reported series of patients managed medically after an ATAI.

\section{CONCLUSIONS}

The advent of thoracic aortic endografting has enabled a revolution in the management of ATAI in patients with multisystem trauma with a low in-hospital morbimortality. Thus, nonoperative management may be only a therapeutic option with acceptable survival in carefully selected patients with multiple severe associated injuries or high-risk 
comorbidities or in patients in whom the aortic repair should be delayed to optimize the results. The natural history of these patients has revealed a marked trend in late aorticrelated complications developing, which may justify an endovascular repair even in some low-risk patients. Moreover, the potential for rapid progression of ATAI in the same patients mandates serial radiologic controls during the first 3 months after injury and diagnosis and then annually.

\section{References}

1. Smith RS, Chang FC. Traumatic rupture of the aorta: still a lethal injury. Am J Surg. 1986;152:660-3.

2. Feczko JD, Lynch L, Pless JE, Clark MA, McClain J, Hawley DA. An autopsy case review of 142 nonpenetrating (blunt) injuries of the aorta. J Trauma. 1992;33:846-9.

3. Crass JR, Cohen AM, Motta AO, Tomashefski JF Jr, Wiesen EJ. A proposed new mechanism of traumatic aortic rupture: the osseous pinch. Radiology. 1990;176: 645-9.

4. Schmoker JD, Lee CH, Taylor RG, Chung A, Trombley L, Hardin N, et al. A novel model of blunt thoracic aortic injury: a mechanism confirmed? J Trauma. 2008;64:923-31.

5. Jamieson WR, Janusz MT, Gudas VM, Burr LH, Fradet GJ, Henderson C. Traumatic rupture of the thoracic aorta: third decade of experience. Am J Surg. 2002; 183:571-5.

6. Fabian TC, Davis KA, Gavant ML, Croce MA, Melton SM, Patton JH Jr, et al. Prospective study of blunt aortic injury: helical CT is diagnostic and antihypertensive therapy reduces rupture. Ann Surg. 1998;227:666-77.

7. Pate JW, Gavant ML, Weiman DS, Fabian TC. Traumatic rupture of the aortic isthmus: program of selective management. World J Surg. 1999;23: 59-63.

8. Baker SP, O'Neill B, Haddon W Jr, Long WB. The injury severity score: a method for describing patients with multiple injuries and evaluating emergency care. J Trauma. 1974;14:187-96.

9. Champion HR, Sacco WJ, Copes WS, Gann DS, Gennarelli TA, Flanagan ME. A revision of the Trauma Score. J Trauma. 1989;29:623-9.

10. Champion HR, Sacco WJ, Copes WS. Injury severity scoring again. J Trauma. 1995;38:94-5.

11. Mosquera VX, Herrera JM, Marini M, Estevez F, Cao I, Gulias D, et al. Mid-term results of thoracic endovascular aortic repair in surgical high-risk patients. Interact Cardiovasc Thorac Surg. 2009;9:61-5.

12. Parmley LF, Mattingly TW, Manion WC, Jahnke EJ Jr. Nonpenetrating traumatic injury of the aorta. Circulation. 1958;17:1086-101.
13. Mattox KL, Wall MJ Jr. Historical review of blunt injury to the thoracic aorta Chest Surg Clin N Am. 2000;10:167-82. x.

14. Demetriades D, Velmahos GC, Scalea TM, Jurkovich GJ, Karmy-Jones R, Teixeira PG, et al. Blunt traumatic thoracic aortic injuries: early or delayed repair-results of an American Association for the Surgery of Trauma prospective study. J Trauma. 2009;66:967-73.

15. Erbel R, Alfonso F, Boileau C, Dirsch O, Eber B, Haverich A, et al. Diagnosis and management of aortic dissection. Eur Heart J. 2001;22:1642-81.

16. Hemmila MR, Arbabi S, Rowe SA, Brandt MM, Wang SC, Taheri PA, et al. Delayed repair for blunt thoracic aortic injury: is it really equivalent to early repair? J Trauma. 2004;56:13-23.

17. Holmes JH, Bloch RD, Hall RA, Carter YM, Karmy-Jones RC. Natural history of traumatic rupture of the thoracic aorta managed nonoperatively: a longitudinal analysis. Ann Thorac Surg. 2002;73:1149-54.

18. Kepros J, Angood P, Jaffe CC, Rabinovici R. Aortic intimal injuries from blun trauma: resolution profile in nonoperative management. J Trauma. 2002;52:475-8

19. Hirose H, Gill IS, Malangoni MA. Nonoperative management of traumatic aortic injury. J Trauma. 2006;60:597-601.

20. Caffarelli AD, Mallidi HR, Maggio PM, Spain DA, Miller DC, Mitchell RS Early outcomes of deliberate nonoperative management for blunt thoracic aortic injury in trauma. J Thorac Cardiovasc Surg. 2010;140:598-605.

21. Pate JW, Fabian TC, Walker W. Traumatic rupture of the aortic isthmus: an emergency? World J Surg. 1995;19:119-26.

22. Akowuah E, Angelini G, Bryan AJ. Open versus endovascular repair of traumatic aortic rupture: a systematic review. J Thorac Cardiovasc Surg. 2009; 138:768-9.

23. Botta L, Russo V, Savini C, Buttazzi K, Pacini D, Lovato L, et al. Endovascular treatment for acute traumatic transection of the descending aorta: focus on operative timing and left subclavian artery management. $J$ Thorac Cardiovasc Surg. 2008;136:1558-63.

24. Sze DY, Mitchell RS, Miller DC, Fleischmann D, Frisoli JK, Kee ST, et al. Infolding and collapse of thoracic endoprostheses: manifestations and treatment options. J Thorac Cardiovasc Surg. 2009;138:324-33.

25. Xenos ES, Abedi NN, Davenport DL, Minion DJ, Hamdallah O, Sorial EE, et al Meta-analysis of endovascular vs open repair for traumatic descending thoracic aortic rupture. J Vasc Surg. 2008;48:1343-51.

26. Svensson LG, Kouchoukos NT, Miller DC, Bavaria JE, Coselli JS, Curi MA et al. Expert consensus document on the treatment of descending thoracic aortic disease using endovascular stent-grafts. Ann Thorac Surg. 2008;85(1 Suppl) S1-41.

27. Hiratzka LF, Bakris GL, Beckman JA, Bersin RM, Carr VF, Casey D Jr, et al 2010 ACCF/AHA/AATS/ACR/ASA/SCA/SCAI/SIR/STS/SVM Guidelines for the Diagnosis and Management of Patients with Thoracic Aortic Disease. J Am Coll Cardiol. 2010;55:e27-129.

28. Steenburg SD, Ravenel JG. Acute traumatic thoracic aortic injuries: experience with 64-MDCT. AJR Am J Roentgenol. 2008;191:1564-9. 